\title{
Theoretical Analysis of the Reaction Mechanism of Biotin Carboxylase
}

\author{
Yuko Ito, ${ }_{1}^{1}$ Hiroki Kondo, ${ }^{* 1}$ Yoshihito Shiota, ${ }^{2}$ and Kazunari Yoshizawa ${ }^{*, 2}$ \\ Department of Bioscience and Bioinformatics, Kyushu Institute of Technology, 680-4 Kawazu, \\ Iizuka 820-8502, Japan, and Institute for Materials Chemistry and Engineering, Kyushu University, \\ Fukuoka 812-8581, Japan
}

E-mail: kondo@bio.kyutech.ac.jp (H.K.); kazunari@ms.ifoc.kyushu-u.ac.jp (K.Y.)

${ }^{1}$ Kyushu Institute of Technology

${ }^{2}$ Kyushu University 


\section{Reference}

(25) Frisch, M. J.; Trucks, G. W.; Schlegel, H. B.; Scuseria, G. E.; Robb, M. A.; Cheeseman, J. R.; Montgomery, J. A., Jr.; Vreven, T.; Kudin, K. N.; Burant, J. C.; Millam, J. M.; Iyengar, S. S.;

Tomasi, J.; Barone, V.; Mennucci, B.; Cossi, M.; Scalmani, G.; Rega, N.; Petersson, G. A.;

Nakatsuji, H.; Hada, M.; Ehara, M.; Toyota, K.; Fukuda, R.; Hasegawa, J.; Ishida, M.; Nakajima, T.; Honda, Y.; Kitao, O.; Nakai, H.; Klene, M.; Li, X.; Knox, J. E.; Hratchian, H. P.; Cross, J. B.; Adamo, C.; Jaramillo, J.; Gomperts, R.; Stratmann, R. E.; Yazyev, O.; Austin, A. J.; Cammi, R.; Pomelli, C.; Ochterski, J. W.; Ayala, P. Y.; Morokuma, K.; Voth, G. A.; Salvador, P.; Dannenberg, J. J.; Zakrzewski, V. G.; Dapprich, S.; Daniels, A. D.; Strain, M. C.; Farkas, O.; Malick, D. K.;

Rabuck, A. D.; Raghavachari, K.; Foresman, J. B.; Ortiz, J. V.; Cui, Q.; Baboul, A. G.; Clifford, S.; Cioslowski, J.; Stefanov, B. B.; Liu, G.; Liashenko, A.; Piskorz, P.; Komaromi, I.; Martin, R. L.; Fox, D. J.; Keith, T.; Al-Laham, M. A.; Peng, C. Y.; Nanayakkara, A.; Challacombe, M.; Gill, P. M. W.; Johnson, B.; Chen, W.; Wong, M. W.; Gonzalez, C.; Pople, J. A. Gaussian 03; Gaussian, Inc.: Wallingford, CT, 2004.

Table S1. Cartesian coordinates of the optimized geometry for the first and second step of the BC reaction.

$\begin{array}{cccc}\text { ATP complex } & & \\ \mathrm{P} & -0.220077 & -1.694044 & -0.529829 \\ \mathrm{P} & 0.244617 & 0.053357 & 1.742574 \\ \mathrm{P} & -0.257568 & 2.719785 & 0.218750 \\ \mathrm{Mg} & 2.233403 & -1.680677 & 0.050851 \\ \mathrm{O} & 4.152873 & -1.254466 & -0.821130 \\ \mathrm{O} & 3.858719 & -2.965896 & 0.563340 \\ \mathrm{O} & 0.853569 & -1.002125 & -1.388693 \\ \mathrm{O} & 0.488853 & -2.846619 & 0.199635 \\ \mathrm{O} & -0.756369 & -0.619750 & 0.610143 \\ \mathrm{O} & -0.252267 & -0.185922 & 3.120353 \\ \mathrm{O} & 1.697048 & -0.317357 & 1.383107 \\ \mathrm{O} & 0.066786 & 1.662291 & 1.405515 \\ \mathrm{O} & -1.537544 & -2.130709 & -1.258106 \\ \mathrm{O} & -0.936292 & 3.924889 & 0.752504 \\ \mathrm{O} & -0.916443 & 1.936480 & -0.975571 \\ \mathrm{O} & 1.195133 & 3.115810 & -0.376060 \\ \mathrm{O} & -3.495472 & -0.716802 & -1.952827\end{array}$



$\begin{array}{llll}O & -5.313462 & 0.542785 & -1.718470\end{array}$
$\begin{array}{llll}\text { O } & -3.372913 & 1.340044 & -0.962530\end{array}$
$\begin{array}{llll}\text { C } & 4.589696 & -2.244210 & -0.173095\end{array}$
$\begin{array}{llll}\text { C } & 2.067779 & 2.095365 & -0.891960\end{array}$
C $\quad-3.941204 \quad 0.368564 \quad-1.526102$
H $\quad 5.667696 \quad-2.496283 \quad-0.253020$

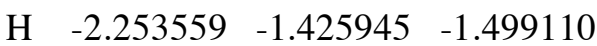
$\begin{array}{llll}\text { H } & 2.543559 & 1.554326 & -0.070087\end{array}$
H $\quad 2.828406 \quad 2.610617 \quad-1.487202$
H $\quad 1.524587 \quad 1.379798 \quad-1.514002$
$\begin{array}{llll}\mathrm{H} & -1.895320 & 1.627039 & -0.903791\end{array}$

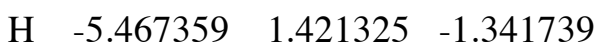

TS1
$\begin{array}{llll}\text { P } & -0.569331 & -1.746265 & 0.017594\end{array}$
$\begin{array}{llll}\text { P } & 0.242859 & 0.398132 & 1.783392\end{array}$
$\begin{array}{llll}\text { P } & -0.326486 & 2.566564 & -0.334929\end{array}$
$\begin{array}{llll}M g & 1.966451 & -1.344028 & -0.179086\end{array}$
$\begin{array}{llll}\text { O } & 3.424960 & -0.875062 & -1.662002\end{array}$
$\begin{array}{llll}\text { O } & 3.915663 & -2.238179 & 0.021586\end{array}$
$\begin{array}{llll}\text { O } & 0.094144 & -1.011828 & -1.178951\end{array}$
$\begin{array}{llll}\text { O } & 0.608356 & -2.650808 & 0.532379\end{array}$
$\begin{array}{llll}\mathrm{O} & -0.859247 & -0.726831 & 1.267484\end{array}$
$\begin{array}{llll}\text { O } & 0.156885 & 0.555239 & 3.255007\end{array}$
$\begin{array}{llll}\text { O } & 1.599002 & 0.107284 & 1.113372\end{array}$
$\begin{array}{llll}\text { O } & -0.353701 & 1.768517 & 1.075836\end{array}$
$\begin{array}{llll}\text { O } & -1.748623 & -2.843778 & -0.137403\end{array}$
$\begin{array}{llll}\text { O } & -1.471127 & 3.492390 & -0.461658\end{array}$
$\begin{array}{llll}\text { O } & -0.031701 & 1.538480 & -1.495701\end{array}$
$\begin{array}{llll}\text { O } & 1.044573 & 3.450016 & -0.288746\end{array}$
$\begin{array}{llll}\text { O } & -4.293527 & -1.198680 & 0.203972\end{array}$
$\begin{array}{llll}\text { O } & -4.418503 & 0.050564 & -1.666903\end{array}$
$\begin{array}{llll}\text { O } & -4.293527 & -1.198680 & 0.203972\end{array}$
$\begin{array}{llll}\text { O } & -4.418503 & 0.050564 & -1.666903\end{array}$
$\begin{array}{llll}\text { O } & -2.403032 & -0.520873 & -0.899600\end{array}$
$\begin{array}{llll}\text { C } & 4.231749 & -1.610703 & -1.026886\end{array}$
$\begin{array}{llll}\text { C } & 2.330022 & 2.829743 & -0.368144\end{array}$ 

C $\quad-3.641675-0.623603 \quad-0.682038$
H $\quad 5.268279 \quad-1.710053 \quad-1.411138$
H $\quad-1.308777 \quad-3.643142 \quad 0.199554$
$\begin{array}{llll}\mathrm{H} & 2.529987 & 2.217629 & 0.515079\end{array}$
H $\quad 3.062751 \quad 3.641734 \quad-0.435160$
H $\quad 2.405195 \quad 2.192999 \quad-1.255073$
$\begin{array}{llll}\mathrm{H} & -0.236193 & 0.552517 & -1.380361\end{array}$
H $\quad-5.310898 \quad-0.098206 \quad-1.323451$

Int 1
$\begin{array}{llll}\mathrm{P} & -0.770840 & -1.644198 & -0.173662\end{array}$
$\begin{array}{llll}\text { P } & 0.090024 & 0.418402 & 1.738292\end{array}$
$\begin{array}{llll}\mathrm{P} & -0.172062 & 2.601453 & -0.433259\end{array}$
$\begin{array}{llll}M g & 1.836659 & -1.362588 & -0.114234\end{array}$
$\begin{array}{llll}\text { O } & 3.436613 & -0.895010 & -1.469531\end{array}$
$\begin{array}{llll}\text { O } & 3.750593 & -2.339079 & 0.190549\end{array}$
$\begin{array}{llll}\text { O } & 0.110830 & -0.904985 & -1.262978\end{array}$
$\begin{array}{llll}\text { O } & 0.386246 & -2.576894 & 0.435755\end{array}$
$\begin{array}{llll}\text { O } & -0.999472 & -0.669844 & 1.175193\end{array}$
$\begin{array}{llll}\text { O } & -0.097234 & 0.630987 & 3.195391\end{array}$
$\begin{array}{llll}\text { O } & 1.490310 & 0.095396 & 1.181154\end{array}$
$\begin{array}{llll}\text { O } & -0.415520 & 1.798830 & 0.950324\end{array}$
O $\quad-1.774002-2.926516 \quad-0.458984$
$\begin{array}{llll}\text { O } & -1.225735 & 3.619670 & -0.653399\end{array}$
$\begin{array}{lllll}\text { O } & 0.109408 & 1.567501 & -1.583536\end{array}$
$\begin{array}{llll}\text { O } & 1.252163 & 3.380338 & -0.241663\end{array}$
$\begin{array}{llll}\text { O } & -3.939358 & -1.223706 & 0.548520\end{array}$
$\begin{array}{llll}\text { O } & -4.300718 & 0.031612 & -1.285677\end{array}$
$\begin{array}{llll}\text { O } & -2.256582 & -0.681913 & -0.917024\end{array}$
C $\quad 4.164634-1.682209 \quad-0.803596$
$\begin{array}{llll}\text { C } & 2.497563 & 2.683271 & -0.330221\end{array}$
$\begin{array}{llll}\text { C } & -3.460878 & -0.696667 & -0.447874\end{array}$
H $\quad 5.225903 \quad-1.803142 \quad-1.110893$
H $\quad-1.215477 \quad-3.643040 \quad-0.112087$
H $\quad 2.617456 \quad 1.983538 \quad 0.500405$
$\begin{array}{llll}\text { H } & 3.283338 & 3.446997 & -0.297280\end{array}$
H $\quad 2.569190 \quad 2.124509 \quad-1.268517$ 

$\mathrm{H} \quad-0.014886 \quad 0.536528 \quad-1.447642$
H $\quad-5.140523 \quad-0.006659 \quad-0.806343$

TS2
$\begin{array}{llll}\mathrm{P} & 1.805039 & 0.436632 & -0.529625\end{array}$
$\begin{array}{llll}\mathrm{P} & -0.614746 & -0.474678 & 1.617175\end{array}$
$\begin{array}{llll}\text { P } & -2.250618 & -1.900829 & -0.579604\end{array}$

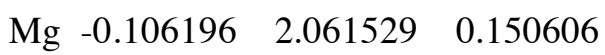
$\begin{array}{llll}\text { O } & -1.166960 & 3.448556 & -1.092240\end{array}$
$\begin{array}{llll}\text { O } & -0.102694 & 4.154747 & 0.726764\end{array}$
$\begin{array}{llll}\text { O } & 0.544643 & 0.623155 & -1.404920\end{array}$
$\begin{array}{llll}\text { O } & 1.862947 & 1.538444 & 0.556220\end{array}$
$\begin{array}{llll}\text { O } & 0.695037 & -0.743462 & 0.818852\end{array}$
$\begin{array}{llll}\text { O } & -0.636447 & -0.835912 & 3.065535\end{array}$
$\begin{array}{llll}\text { O } & -1.215494 & 0.921089 & 1.231173\end{array}$
$\begin{array}{llll}\text { O } & -1.739053 & -1.567013 & 0.886414\end{array}$
$\begin{array}{llll}\text { O } & 3.097533 & 0.969725 & -1.473559\end{array}$
$\begin{array}{llll}\text { O } & -2.317835 & -3.347205 & -0.906493\end{array}$
$\begin{array}{lllll}\text { O } & -1.497320 & -0.986134 & -1.644182\end{array}$
O $\quad-3.774961 \quad-1.299553-0.621392$
$\begin{array}{llll}\text { O } & 4.093929 & -1.037591 & 0.817625\end{array}$
$\begin{array}{lllll}\text { O } & 3.673073 & -2.903830 & -0.381847\end{array}$
O $\quad 2.424288-1.154048 \quad-0.740880$
$\begin{array}{lllll}\text { C } & -0.824913 & 4.358510 & -0.286578\end{array}$
C $\quad-3.954730 \quad 0.101286 \quad-0.401425$
C $3.427181-1.607628 \quad-0.021953$
H $\quad-1.179153 \quad 5.393633 \quad-0.483165$
H $\quad 3.258179 \quad 1.856438 \quad-1.124499$
$\begin{array}{llll}\mathrm{H} & -3.397214 & 0.445751 & 0.474911\end{array}$
$\mathrm{H} \quad-5.028603 \quad 0.264254 \quad-0.251276$
H $\quad-3.619569 \quad 0.676659-1.272616$
H $\quad-0.654531 \quad-0.488371 \quad-1.420859$
H $\quad 4.389393 \quad-3.164347 \quad 0.214236$

Carboxyphosphate complex
$\begin{array}{llll}\text { P } & 2.936375 & -0.872122 & 0.347671\end{array}$
$\begin{array}{llll}\mathrm{P} & -2.261500 & 1.017122 & -0.806560\end{array}$ 
Biotin complex
$\begin{array}{llll}\mathrm{P} & -2.382904 & -0.009673 & 0.797863\end{array}$
$\begin{array}{llll}\text { O } & -1.609859 & 1.004251 & 0.015529\end{array}$
$\begin{array}{llll}O & -3.089055 & 0.205073 & 2.092700\end{array}$
$\begin{array}{llll}\text { O } & -1.439532 & -1.367539 & 0.929671\end{array}$
$\begin{array}{llll}\text { C } & -4.527040 & -0.083282 & -0.801377\end{array}$
$\begin{array}{llll}\text { O } & -5.232914 & -0.907355 & -1.638363\end{array}$
H $\quad 0.628324 \quad-1.793135 \quad-0.368714$
$\begin{array}{llll}\text { O } & -3.500120 & -0.755054 & -0.316982\end{array}$ 

C $\quad 2.086026 \quad 2.205216 \quad-0.174306$
$\begin{array}{llll}\mathrm{N} & 1.152189 & 1.208756 & -0.162184\end{array}$
$\begin{array}{llll}\text { C } & 1.645745 & -0.024208 & 0.413297\end{array}$
$\begin{array}{llll}\text { C } & 3.154948 & 0.287556 & 0.728142\end{array}$
$\begin{array}{llll}\mathrm{N} & 3.325183 & 1.619821 & 0.174384\end{array}$
O $\quad 1.945946 \quad 3.386114 \quad-0.455075$
H $\quad 3.985311 \quad 2.261856 \quad 0.587278$
$\begin{array}{llll}\text { C } & 1.559295 & -1.245272 & -0.519729\end{array}$
S $\quad 2.989861 \quad-2.304006 \quad-0.059086$
$\begin{array}{llll}\text { C } & 4.076075 & -0.809915 & 0.103950\end{array}$
$\begin{array}{llll}\text { C } & 4.721471 & -0.416458 & -1.226784\end{array}$
H $\quad 5.322241 \quad-1.245879 \quad-1.611158$
H $\quad 5.366283 \quad 0.459706 \quad-1.101838$
H $\quad 3.969228 \quad-0.156881 \quad-1.975766$
H $\quad-5.956476 \quad-0.344664 \quad-1.950474$
H $\quad 1.634677 \quad-0.937565 \quad-1.566275$
$\begin{array}{llll}\text { O } & -4.866481 & 1.062701 & -0.596231\end{array}$
$\begin{array}{llll}\mathrm{H} & 0.135634 & 1.375330 & -0.182687\end{array}$
H $\quad-1.758850 \quad-1.839576 \quad 1.710696$
$\begin{array}{llll}\text { H } & 3.311570 & 0.277286 & 1.814817\end{array}$
$\begin{array}{llll}\mathrm{H} & 1.101971 & -0.270671 & 1.330369\end{array}$
$\begin{array}{llll}\text { H } & 4.861253 & -1.083993 & 0.816617\end{array}$

TS3
$\begin{array}{llll}\mathrm{P} & 2.249853 & -1.137694 & -0.399047\end{array}$
$\begin{array}{llll}\text { O } & 1.755884 & -1.151980 & 1.125822\end{array}$
$\begin{array}{llll}\text { O } & 1.187951 & -0.964365 & -1.436657\end{array}$
$\begin{array}{llll}\text { O } & 2.921982 & -2.617425 & -0.501122\end{array}$
$\begin{array}{llll}\text { C } & 3.540947 & 1.218025 & 0.085303\end{array}$
$\begin{array}{llll}\text { O } & 2.524832 & 1.472828 & 0.783384\end{array}$
$\begin{array}{llll}\mathrm{H} & -3.050443 & 0.201834 & 2.838029\end{array}$
$\begin{array}{llll}\text { O } & 3.520520 & -0.179182 & -0.501197\end{array}$
$\begin{array}{llll}\text { C } & -0.494760 & 1.322372 & 0.068154\end{array}$
$\begin{array}{llll}\mathrm{N} & -0.567730 & 0.806220 & 1.251683\end{array}$
$\begin{array}{llll}\text { C } & -1.653698 & -0.171159 & 1.171522\end{array}$
$\begin{array}{llll}\text { C } & -1.893511 & -0.415775 & -0.348911\end{array}$
$\begin{array}{llll}\text { N } & -1.395874 & 0.854237 & -0.897222\end{array}$ 

$\begin{array}{llll}\mathrm{O} & 0.349384 & 2.258280 & -0.333096\end{array}$
H $\quad-0.934116 \quad 0.768365 \quad-1.797175$
$\begin{array}{llll}\text { C } & -2.958904 & 0.407506 & 1.768757\end{array}$
$\begin{array}{llll}S & -4.366125 & -0.382652 & 0.890598\end{array}$
$\begin{array}{llll}\text { C } & -3.370900 & -0.758919 & -0.650095\end{array}$
$\begin{array}{llll}\text { C } & -3.945237 & -0.071802 & -1.888698\end{array}$

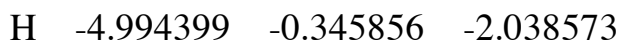
H $\quad-3.386894 \quad-0.373371 \quad-2.784515$
$\begin{array}{llll}\mathrm{H} & -3.873441 & 1.013313 & -1.792461\end{array}$
H $\quad 1.205662 \quad 2.139322 \quad 0.185260$
H $\quad-2.980409 \quad 1.489326 \quad 1.618300$
$\begin{array}{llll}\text { O } & 4.543883 & 1.826088 & -0.209892\end{array}$
$\begin{array}{llll}\mathrm{H} & 1.531211 & -0.228399 & 1.380401\end{array}$
H $\quad 2.540534 \quad-3.033261 \quad-1.285814$
$\begin{array}{llll}\mathrm{H} & -1.248497 & -1.227551 & -0.706312\end{array}$
$\begin{array}{llll}\text { H } & -1.390902 & -1.099811 & 1.687398\end{array}$
H $\quad-3.445978 \quad-1.843254 \quad-0.778450$

Int 2
P $\quad-2.634698 \quad-1.303589 \quad-0.271244$
$\begin{array}{llll}\text { O } & -3.273514 & -1.065326 & -1.686799\end{array}$
$\begin{array}{llll}\text { O } & -1.329360 & -2.022195 & -0.172224\end{array}$
$\begin{array}{llll}\text { O } & -3.781622 & -2.107165 & 0.565186\end{array}$
$\begin{array}{llll}\text { C } & -2.837378 & 1.437441 & -0.244123\end{array}$
$\begin{array}{llll}\text { O } & -2.428615 & 2.394881 & 0.408270\end{array}$
H $\quad 3.981432 \quad 2.222290 \quad-1.055558$
$\begin{array}{llll}\text { O } & -2.590858 & 0.153926 & 0.422944\end{array}$
$\begin{array}{llll}\text { C } & 0.587887 & 1.074429 & 0.899793\end{array}$
$\begin{array}{llll}\mathrm{N} & 1.215199 & 1.901351 & 0.134013\end{array}$
$\begin{array}{llll}\text { C } & 2.122311 & 1.093638 & -0.674119\end{array}$
$\begin{array}{llll}\text { C } & 1.867877 & -0.413739 & -0.279544\end{array}$
$\begin{array}{llll}\mathrm{N} & 0.937869 & -0.278090 & 0.836215\end{array}$
$\begin{array}{llll}\text { O } & -0.349896 & 1.386867 & 1.795112\end{array}$
$\begin{array}{llll}\mathrm{H} & 0.136735 & -0.910564 & 0.811551\end{array}$
$\begin{array}{llll}\text { C } & 3.602253 & 1.429611 & -0.406348\end{array}$
S $\quad 4.540535 \quad-0.115353 \quad-0.738228$ 

$\begin{array}{llll}\text { C } & 3.209486 & -1.137419 & 0.065261\end{array}$
$\begin{array}{llll}\text { C } & 3.464737 & -1.333826 & 1.560962\end{array}$
$\mathrm{H} \quad 4.425244-1.834800 \quad 1.717275$
H $\quad 2.668900-1.937916 \quad 2.007152$
$\mathrm{H} \quad 3.481022 \quad-0.379278 \quad 2.093489$

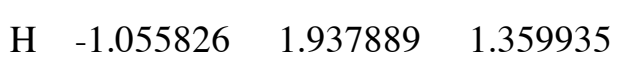
H $3.733993 \quad 1.739841 \quad 0.634372$
$\begin{array}{llll}\text { O } & -3.423476 & 1.369866 & -1.350629\end{array}$
H $\quad-3.442510 \quad-0.025161 \quad-1.732245$
$\mathrm{H} \quad-3.329942 \quad-2.720259 \quad 1.160757$
$\mathrm{H} \quad 1.387581 \quad-0.954231 \quad-1.101661$
$\mathrm{H} \quad 1.927603 \quad 1.246839 \quad-1.743120$
H $\quad 3.245526 \quad-2.110356 \quad-0.434587$

TS4B
$\begin{array}{llll}\text { P } & 5.197028 & 0.186625 & 0.057432\end{array}$
$\begin{array}{llll}\text { O } & 4.873258 & -0.695479 & 1.291152\end{array}$
$\begin{array}{llll}\text { O } & 6.343917 & -0.109270 & -0.836258\end{array}$
$\begin{array}{llll}\text { O } & 5.283842 & 1.714507 & 0.636543\end{array}$
C $\quad 2.570478-0.344913-0.378056$
$\begin{array}{llll}\text { O } & 1.617126 & -0.166707 & -1.145418\end{array}$
$\mathrm{H} \quad-3.765121 \quad 2.710010 \quad-0.576037$
$\begin{array}{llll}\text { O } & 3.775881 & 0.220460 & -0.804022\end{array}$
$\begin{array}{llll}\text { C } & -1.572506 & -0.601583 & -0.135067\end{array}$
$\begin{array}{llll}\mathrm{N} & -1.640840 & 0.682982 & -0.017117\end{array}$
$\begin{array}{llll}\text { C } & -2.964495 & 0.969747 & 0.513044\end{array}$
$\begin{array}{llll}\text { C } & -3.608508 & -0.408861 & 0.926410\end{array}$
$\begin{array}{llll}\mathrm{N} & -2.724660 & -1.329055 & 0.222767\end{array}$
$\begin{array}{llll}\text { O } & -0.575769 & -1.333513 & -0.571193\end{array}$
$\mathrm{H} \quad-2.540596 \quad-2.235858 \quad 0.632189$
$\begin{array}{lllll}\text { C } & -3.888937 & 1.625768 & -0.531437\end{array}$
$\begin{array}{llll}\text { S } & -5.601991 & 1.239995 & 0.002196\end{array}$
$\begin{array}{llll}\text { C } & -5.114584 & -0.484350 & 0.516495\end{array}$
$\begin{array}{llll}\text { C } & -5.431590 & -1.513278 & -0.570779\end{array}$
H $\quad-6.499543 \quad-1.494489-0.808264$
H $\quad-5.168751 \quad-2.524133 \quad-0.237226$
H $\quad-4.865742 \quad-1.314638 \quad-1.483181$ 

H $\quad 0.276863 \quad-0.791099 \quad-0.756418$

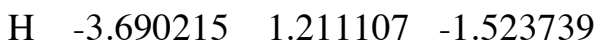
$\begin{array}{llll}\text { O } & 2.572007 & -0.981441 & 0.725914\end{array}$
H $\quad 3.754593 \quad-0.931377 \quad 1.172394$
H $\quad 5.840493 \quad 2.221885 \quad 0.030483$
$\begin{array}{llll}\mathrm{H} & -3.539261 & -0.546356 & 2.014469\end{array}$

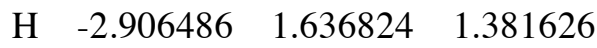
$\begin{array}{llll}\mathrm{H} & -5.722525 & -0.702308 & 1.400150\end{array}$

Int 3B
$\begin{array}{llll}\text { P } & 5.082187 & 0.270622 & 0.127383\end{array}$
$\begin{array}{llll}\text { O } & 4.551366 & 0.183308 & 1.555622\end{array}$
$\begin{array}{llll}\text { O } & 6.205247 & -0.536391 & -0.401412\end{array}$
$\begin{array}{llll}\text { O } & 5.301587 & 1.854183 & -0.226334\end{array}$
C $\quad 2.523263 \quad-0.524598 \quad-0.423950$
$\begin{array}{llll}\text { O } & 1.640124 & -0.756431 & -1.250383\end{array}$
H $\quad-3.471555 \quad 2.640368 \quad-0.875179$
$\begin{array}{llll}\text { O } & 3.698501 & -0.030281 & -0.855146\end{array}$
$\begin{array}{llll}\text { C } & -1.535174 & -0.816819 & -0.080359\end{array}$
$\begin{array}{llll}\mathrm{N} & -1.507705 & 0.468143 & -0.195184\end{array}$
$\begin{array}{llll}\text { C } & -2.763426 & 0.954401 & 0.355754\end{array}$
$\begin{array}{llll}\text { C } & -3.513334 & -0.288379 & 0.979639\end{array}$
$\begin{array}{llll}\text { N } & -2.707534 & -1.381678 & 0.452945\end{array}$
$\begin{array}{lllll}\text { O } & -0.612708 & -1.687234 & -0.424091\end{array}$
H $\quad-2.565486 \quad-2.205369 \quad 1.022653$
$\begin{array}{llll}\text { C } & -3.682327 & 1.581538 & -0.710548\end{array}$
$\begin{array}{llll}\text { S } & -5.390900 & 1.403486 & -0.061234\end{array}$
$\begin{array}{llll}\text { C } & -5.018467 & -0.311275 & 0.559658\end{array}$
$\begin{array}{llll}\text { C } & -5.378837 & -1.380921 & -0.473449\end{array}$
Н $\quad-6.441890 \quad-1.317749 \quad-0.724178$
H $\quad-5.169369-2.383198 \quad-0.083939$
$\begin{array}{llll}\mathrm{H} & -4.796727 & -1.262815 & -1.390134\end{array}$

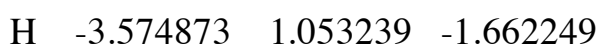
H $\quad 2.367953 \quad-0.735769 \quad 0.862734$
$\begin{array}{llll}\text { O } & 3.233410 & -0.408031 & 1.342844\end{array}$
H $\quad 4.747442 \quad 2.368636 \quad 0.376470$
$\begin{array}{llll}\mathrm{H} & -3.462387 & -0.248088 & 2.075656\end{array}$ 

$\mathrm{H} \quad-2.582184 \quad 1.710663 \quad 1.129548$
$\begin{array}{llll}\mathrm{H} & -5.648216 & -0.443221 & 1.445288\end{array}$
H $\quad 0.253358 \quad-1.239562 \quad-0.720503$

\section{TS5B}
$\begin{array}{llll}\text { P } & -2.927130 & -0.977684 & 0.082440\end{array}$
$\begin{array}{llll}\text { O } & -2.431870 & -0.845048 & 1.508809\end{array}$
$\begin{array}{llll}\text { O } & -4.351291 & -1.119437 & -0.321963\end{array}$
$\begin{array}{llll}\text { O } & -2.022420 & -2.141355 & -0.643628\end{array}$
$\begin{array}{llll}\text { C } & -1.661164 & 1.406708 & -0.140171\end{array}$
$\begin{array}{llll}\text { O } & -1.574918 & 2.489672 & -0.864558\end{array}$
H $\quad 0.416336 \quad-2.139140 \quad-0.700532$
$\begin{array}{llll}O & -2.305449 & 0.400506 & -0.737371\end{array}$
C $\quad 1.088476 \quad 1.934286 \quad-0.114405$
$\begin{array}{llll}\mathrm{N} & 0.319631 & 0.863950 & -0.285117\end{array}$
$\begin{array}{llll}\text { C } & 0.954083 & -0.282832 & 0.336054\end{array}$
$\begin{array}{llll}\text { C } & 2.326111 & 0.245964 & 0.918750\end{array}$
$\begin{array}{llll}\mathrm{N} & 2.351225 & 1.615810 & 0.426113\end{array}$
$\begin{array}{llll}\text { O } & 0.780540 & 3.132912 & -0.377576\end{array}$
H $2.755030 \quad 2.344062 \quad 0.999023$
$\begin{array}{llll}\text { C } & 1.245055 & -1.432681 & -0.647188\end{array}$
$\begin{array}{llll}\text { S } & 2.742908 & -2.278727 & 0.001567\end{array}$
C $3.513199 \quad-0.647073 \quad 0.437480$
C $4.319125 \quad-0.064639-0.726322$
H $\quad 5.112764 \quad-0.759380 \quad-1.017583$
H $\quad 4.770983 \quad 0.892886 \quad-0.446264$
$\begin{array}{llll}\text { H } & 3.685079 & 0.121840 & -1.596623\end{array}$
H $\quad-0.653782 \quad 2.944010 \quad-0.661402$
$\begin{array}{llll}\text { H } & 1.438060 & -1.033271 & -1.647044\end{array}$
$\begin{array}{llll}\text { O } & -1.746609 & 1.533920 & 1.170268\end{array}$
$\begin{array}{llll}\mathrm{H} & -1.984054 & 0.602645 & 1.535073\end{array}$
$\begin{array}{llll}\mathrm{H} & -2.527639 & -2.459995 & -1.403924\end{array}$
$\begin{array}{llll}\mathrm{H} & 2.301611 & 0.214052 & 2.015580\end{array}$
$\mathrm{H} \quad 0.326681 \quad-0.678841 \quad 1.143675$
H $\quad 4.190168 \quad-0.850061 \quad 1.274361$

Int 4B 

$\begin{array}{llll}\text { P } & 3.024912 & -0.690712 & 0.097210\end{array}$
$\begin{array}{llll}\text { O } & 3.561451 & 0.207487 & -0.996458\end{array}$
$\begin{array}{llll}\text { O } & 3.811531 & -1.134856 & 1.284563\end{array}$
$\begin{array}{llll}\text { O } & 2.386888 & -2.058801 & -0.613971\end{array}$
$\begin{array}{llll}\text { C } & 1.020813 & 1.175183 & 0.120708\end{array}$
$\begin{array}{llll}\text { O } & 1.330380 & 2.205705 & 0.996201\end{array}$
H $\quad-0.062668 \quad-2.151931-0.076927$
$\begin{array}{llll}\text { O } & 1.580419 & 0.000988 & 0.628805\end{array}$
$\begin{array}{llll}\text { C } & -1.308626 & 2.020882 & -0.016292\end{array}$
$\begin{array}{llll}\mathrm{N} & -0.456273 & 0.946848 & 0.137651\end{array}$
$\begin{array}{llll}\text { C } & -0.963744 & -0.222553 & -0.581546\end{array}$
$\begin{array}{llll}\text { C } & -2.455242 & 0.150589 & -0.894226\end{array}$
N $\quad-2.521928 \quad 1.540758 \quad-0.486877$
$\begin{array}{llll}\text { O } & -1.084864 & 3.205142 & 0.234284\end{array}$
$\mathrm{H} \quad-3.162118 \quad 2.208146 \quad-0.886058$
$\begin{array}{llll}\text { C } & -0.908821 & -1.534294 & 0.222601\end{array}$
S $\quad-2.489401 \quad-2.415299 \quad-0.135348$
C $\quad-3.411685-0.813234-0.132762$
C $\quad-3.788541 \quad-0.349063 \quad 1.275870$
H $\quad-4.414344 \quad-1.100675 \quad 1.764927$
$\mathrm{H} \quad-4.337942 \quad 0.597333 \quad 1.237680$
H $\quad-2.901350 \quad-0.185714 \quad 1.893357$
$\begin{array}{llll}\mathrm{H} & 0.747418 & 2.946674 & 0.741209\end{array}$
H $\quad-0.824329 \quad-1.322418 \quad 1.289554$
O $1.356362 \quad 1.446651 \quad-1.189339$
H $2.675332-2.782176-0.041285$
H $\quad-2.648074 \quad 0.037105 \quad-1.966977$
H $\quad-0.396412-0.339145 \quad-1.510155$
$\mathrm{H} \quad-4.322003 \quad-0.977537 \quad-0.719800$
H $\quad 2.308377 \quad 1.118983 \quad-1.292309$

TS6B
$\begin{array}{llll}\text { P } & -2.953012 & -0.724204 & -0.372078\end{array}$
$\begin{array}{llll}\text { O } & -3.369475 & -0.648459 & 1.132663\end{array}$
$\begin{array}{llll}\text { O } & -3.986759 & -0.538493 & -1.428010\end{array}$
$\begin{array}{lllll}\text { O } & -2.274665 & -2.223196 & -0.561067\end{array}$
$\begin{array}{llll}\text { C } & -1.158509 & 1.103093 & 0.672708\end{array}$ 

$\begin{array}{llll}\mathrm{O} & -1.662242 & 2.375922 & 0.463514\end{array}$
$\mathrm{H} \quad 0.196843 \quad-1.915018 \quad 0.447895$
$\begin{array}{lllll}\mathrm{O} & -1.661932 & 0.242005 & -0.505402\end{array}$
$\begin{array}{llll}\text { C } & 0.946579 & 1.855417 & -0.530412\end{array}$
$\begin{array}{llll}\mathrm{N} & 0.310045 & 1.116612 & 0.429176\end{array}$
$\begin{array}{llll}\text { C } & 1.155518 & 0.022388 & 0.874692\end{array}$
$\begin{array}{llll}\text { C } & 2.569001 & 0.577258 & 0.570093\end{array}$
$\begin{array}{llll}\mathrm{N} & 2.272077 & 1.393162 & -0.607081\end{array}$
$\begin{array}{llll}\text { O } & 0.491631 & 2.771232 & -1.214612\end{array}$
H $2.934999 \quad 2.093492-0.910830$
$\begin{array}{llll}\text { C } & 0.978477 & -1.270123 & 0.047528\end{array}$
S $\quad 2.596530 \quad-2.140050 \quad 0.117824$
$\begin{array}{llll}\text { C } & 3.580076 & -0.573406 & 0.357325\end{array}$
$\begin{array}{llll}\text { C } & 4.582554 & -0.334757 & -0.772992\end{array}$
$\mathrm{H} \quad 5.270155 \quad-1.180535 \quad-0.865611$
$\mathrm{H} \quad 5.184721 \quad 0.563360 \quad-0.576579$
H $\quad 4.064278-0.204848 \quad-1.725590$
H $\quad-1.280992 \quad 2.699292 \quad-0.375019$
$\mathrm{H} \quad 0.720540 \quad-1.028104 \quad-0.983361$
$\begin{array}{llll}\text { O } & -1.435295 & 0.590479 & 1.830328\end{array}$
$\mathrm{H} \quad-2.648043 \quad-2.575993 \quad-1.379337$
$\mathrm{H} \quad 2.920963 \quad 1.207998 \quad 1.401872$
$\mathrm{H} \quad 0.992303 \quad-0.174060 \quad 1.934405$
H $\quad 4.130900 \quad-0.707974 \quad 1.294040$
H $\quad-2.543092 \quad-0.077324 \quad 1.626474$

\section{TS4C}
P $\quad-2.373125 \quad-1.236696-0.133605$
$\begin{array}{llll}\text { O } & -1.991959 & -0.984681 & -1.699411\end{array}$
$\begin{array}{llll}\text { O } & -1.342956 & -2.123822 & 0.513833\end{array}$
$\begin{array}{llll}\mathrm{O} & -3.778837 & -2.095744 & -0.214032\end{array}$
$\begin{array}{llll}\text { C } & -2.554459 & 1.906647 & -0.338969\end{array}$
$\begin{array}{llll}\text { O } & -2.451661 & 2.601394 & 0.620697\end{array}$
H $\quad 3.256914 \quad 2.108214 \quad-1.509703$
$\begin{array}{llll}\text { O } & -2.716381 & 0.127162 & 0.478108\end{array}$
$\begin{array}{llll}\text { C } & 0.412386 & 0.887132 & 1.145938\end{array}$
$\begin{array}{llll}\mathrm{N} & 0.791431 & 1.685306 & 0.206183\end{array}$ 

C $\quad 1.620266 \quad 0.876706 \quad-0.684975$
$\begin{array}{llll}\text { C } & 1.591943 & -0.592770 & -0.113872\end{array}$
$\begin{array}{llll}\mathrm{N} & 0.871159 & -0.424016 & 1.149923\end{array}$
$\begin{array}{llll}\text { O } & -0.394491 & 1.223150 & 2.160346\end{array}$
H $\quad 0.109728 \quad-1.105019 \quad 1.272033$
$\begin{array}{llll}\text { C } & 3.081383 & 1.362872 & -0.730237\end{array}$
S $4.099118 \quad-0.124809 \quad-1.087303$
$\begin{array}{llll}\text { C } & 3.036090 & -1.169726 & 0.027633\end{array}$
$\begin{array}{llll}\text { C } & 3.574999 & -1.206730 & 1.459176\end{array}$
H $\quad 4.591951 \quad-1.611596 \quad 1.469771$
H $\quad 2.934437 \quad-1.829459 \quad 2.090965$
H $\quad 3.595403 \quad-0.208938 \quad 1.905705$
H $\quad-1.018879 \quad 1.891331 \quad 1.819504$
$\begin{array}{llll}\mathrm{H} & 3.363197 & 1.795808 & 0.234219\end{array}$
$\begin{array}{llll}\text { O } & -2.641879 & 1.736023 & -1.518127\end{array}$
H $\quad-2.241747 \quad-0.068198 \quad-1.923646$
$\begin{array}{llll}\mathrm{H} & -3.555265 & -2.982444 & 0.098727\end{array}$
H $\quad 1.011628 \quad-1.247260 \quad-0.770534$

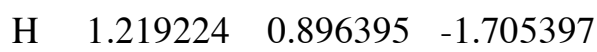
$\begin{array}{llll}\text { H } & 3.074461 & -2.178500 & -0.394763\end{array}$

Int 3C
P $\quad-2.374841 \quad-1.272943 \quad-0.074934$
O
$\begin{array}{llll}\mathrm{O} & -1.470888 & -2.161130 & 0.754288\end{array}$
$\begin{array}{llll}\text { O } & -3.716880 & -2.182264 & -0.419219\end{array}$
$\begin{array}{lllll}\text { C } & -2.088233 & 2.351492 & -0.501383\end{array}$
$\begin{array}{llll}\text { O } & -2.240769 & 2.872986 & 0.537161\end{array}$
H $\quad 3.010132 \quad 2.058715 \quad-1.580439$
$\begin{array}{llll}\text { O } & -2.811926 & 0.099869 & 0.368390\end{array}$
$\begin{array}{llll}\text { C } & 0.308800 & 0.791777 & 1.238465\end{array}$
$\begin{array}{llll}\mathrm{N} & 0.655101 & 1.618048 & 0.306690\end{array}$
$\begin{array}{llll}\text { C } & 1.433788 & 0.825817 & -0.645089\end{array}$
C $\quad \begin{array}{llll}1.447759 & -0.650143 & -0.089700\end{array}$
$\begin{array}{llll}\mathrm{N} & 0.752273 & -0.515481 & 1.193086\end{array}$
$\begin{array}{llll}\text { O } & -0.458033 & 1.114999 & 2.289883\end{array}$
H $\quad-0.015580 \quad-1.201765 \quad 1.316282$ 

$\begin{array}{llll}\text { C } & 2.884780 & 1.325929 & -0.779462\end{array}$
S $3.901748 \quad-0.155856 \quad-1.165514$
$\begin{array}{llll}\text { C } & 2.906685 & -1.195220 & 0.013677\end{array}$
$\begin{array}{llll}\text { C } & 3.498007 & -1.185098 & 1.424750\end{array}$
H $\quad 4.523184 \quad-1.568504 \quad 1.408779$
H $\quad 2.892710 \quad-1.803939 \quad 2.093667$
H $\quad 3.513618 \quad-0.175848 \quad 1.845566$
$\begin{array}{llll}\mathrm{H} & -1.022081 & 1.851842 & 2.002724\end{array}$
$\begin{array}{llll}\mathrm{H} & 3.213908 & 1.779562 & 0.160193\end{array}$
$\begin{array}{llll}\text { O } & -1.947813 & 2.011172 & -1.614894\end{array}$
H $\quad-1.849763 \quad-0.189653 \quad-1.850436$
H $\quad-3.499656 \quad-3.068679 \quad-0.101145$
H $\quad 0.866124 \quad-1.305031 \quad-0.743226$
H $\quad 0.962349 \quad 0.852893 \quad-1.634602$
H $\quad 2.950756 \quad-2.213168 \quad-0.385666$

TS5C
$\begin{array}{llll}\text { P } & 3.456592 & 0.126171 & 0.150867\end{array}$
$\begin{array}{llll}\text { O } & 5.051906 & 0.151673 & -0.271190\end{array}$
$\begin{array}{llll}\text { O } & 3.291515 & -0.601143 & 1.446697\end{array}$
$\begin{array}{llll}\text { O } & 2.685019 & -0.309793 & -1.097737\end{array}$
$\begin{array}{llll}\text { C } & -3.195026 & 2.725330 & 0.441412\end{array}$
$\begin{array}{llll}\text { O } & -2.292971 & 3.469053 & 0.615864\end{array}$
$\begin{array}{llll}\mathrm{H} & 2.258762 & 1.924137 & 0.155024\end{array}$
$\begin{array}{llll}\text { O } & 3.199102 & 1.755478 & 0.324876\end{array}$
$\begin{array}{llll}\text { C } & -0.582524 & 0.963479 & -0.521070\end{array}$
$\begin{array}{llll}\mathrm{N} & -1.865181 & 0.872640 & -0.306287\end{array}$
$\begin{array}{llll}\text { C } & -2.240268 & -0.464353 & -0.780904\end{array}$
C $\quad-0.879810 \quad-1.183916 \quad-1.130990$
$\begin{array}{llll}\mathrm{N} & 0.060516 & -0.074113 & -1.086564\end{array}$
$\begin{array}{llll}\mathrm{O} & 0.134730 & 2.065413 & -0.223249\end{array}$
H $\quad 1.124277 \quad-0.169950 \quad-1.090717$
$\begin{array}{llll}\text { C } & -3.005993 & -1.300728 & 0.267316\end{array}$
$\begin{array}{llll}\text { S } & -2.326424 & -3.010070 & 0.173735\end{array}$
C $\quad-0.615709 \quad-2.339198 \quad-0.119446$
$\begin{array}{llll}\text { C } & 0.095779 & -1.939817 & 1.173930\end{array}$
$\begin{array}{llll}\mathrm{H} & -0.440174 & -1.143167 & 1.699735\end{array}$ 

H $\quad 1.116978 \quad-1.590999 \quad 0.987326$
H $\quad 0.150273 \quad-2.802191 \quad 1.844733$
$\begin{array}{llll}\mathrm{H} & -0.486828 & 2.704832 & 0.165789\end{array}$
$\begin{array}{llll}\text { O } & -4.253755 & 2.229177 & 0.364549\end{array}$
H $\quad-4.081104 \quad-1.337864 \quad 0.077590$
$\begin{array}{llll}\mathrm{H} & -2.844115 & -0.885707 & 1.264971\end{array}$
H $\quad-0.919448 \quad-1.622100 \quad-2.133586$
H $\quad-2.861626 \quad-0.378772 \quad-1.682836$
H $\quad-0.057187 \quad-3.141399 \quad-0.611453$
H $\quad 5.063691 \quad 0.278400 \quad-1.229367$

Product complex
$\begin{array}{llll}\text { P } & 3.549919 & -0.069222 & -0.064768\end{array}$
$\begin{array}{llll}\text { O } & 4.998124 & -0.316670 & -0.319289\end{array}$
$\begin{array}{llll}\text { O } & 3.192388 & -0.493002 & 1.506295\end{array}$
$\begin{array}{llll}\text { O } & 2.456962 & -0.755804 & -0.895617\end{array}$
$\begin{array}{llll}\text { C } & -2.518455 & 2.236638 & 0.192526\end{array}$
$\begin{array}{llll}\mathrm{O} & -1.823211 & 3.321596 & 0.536185\end{array}$
H $2.300243 \quad 1.729971-0.033790$
$\begin{array}{llll}\text { O } & 3.257495 & 1.540252 & -0.080680\end{array}$
$\begin{array}{llll}\text { C } & -0.334876 & 1.135718 & -0.349826\end{array}$
N $\quad \begin{array}{llll}-1.737972 & 1.137987 & -0.181881\end{array}$
C $\quad-2.324422-0.110721 \quad-0.660380$
C $\quad-1.055631-0.937421 \quad-1.070355$
N $\quad 0.050088 \quad-0.011168 \quad-0.903074$
$\begin{array}{llll}\text { O } & 0.375538 & 2.109244 & -0.011572\end{array}$
H $\quad 1.135827 \quad-0.307964 \quad-0.944749$
$\begin{array}{llll}\text { C } & -3.130406 & -0.880374 & 0.401250\end{array}$
$\begin{array}{llll}\text { S } & -2.737735 & -2.654960 & 0.126612\end{array}$
$\begin{array}{llll}\text { C } & -0.960235 & -2.233787 & -0.206721\end{array}$
$\begin{array}{llll}\text { C } & -0.116345 & -2.104034 & 1.062486\end{array}$
H $\quad-0.486107 \quad-1.311570 \quad 1.720498$
$\begin{array}{llll}\mathrm{H} & 0.921502 & -1.869967 & 0.806920\end{array}$
$\begin{array}{llll}\mathrm{H} & -0.141241 & -3.043821 & 1.621064\end{array}$
$\mathrm{H} \quad-0.848050 \quad 3.111083 \quad 0.430618$
$\begin{array}{llll}\text { O } & -3.733474 & 2.175319 & 0.202177\end{array}$
H $\quad 4.033495 \quad-0.460170 \quad 1.981190$ 

H $\quad-4.203870 \quad-0.719004 \quad 0.304691$
H $\quad-2.823910 \quad-0.577861 \quad 1.405689$
H $\quad-1.138376-1.244338-2.118250$
Н $\quad-2.962199 \quad 0.087987 \quad-1.526649$
$\mathrm{H} \quad-0.565530 \quad-3.047983 \quad-0.820672$

Table S2. The obtained zero-point energy and thermochemical data at the frequency calculation for each transition state in the $\mathrm{BC}$ reaction.

TS1
Zero-point correction $=$
0.159365 (Hartree/Particle)
Thermal correction to Energy=
0.183788
Thermal correction to Enthalpy=
0.184732
Thermal correction to Gibbs Free Energy=
0.102716
Sum of electronic and zero-point Energies=
$-2471.448620$
Sum of electronic and thermal Energies=
$-2471.424196$
Sum of electronic and thermal Enthalpies=
$-2471.423252$
Sum of electronic and thermal Free Energies=
$-2471.505269$

TS2

Zero-point correction= 0.159638 (Hartree/Particle)

Thermal correction to Energy=

0.184156

Thermal correction to Enthalpy=

0.185100

Thermal correction to Gibbs Free Energy=

0.101473

Sum of electronic and zero-point Energies= $-2471.441902$

Sum of electronic and thermal Energies= $-2471.417384$

Sum of electronic and thermal Enthalpies= $-2471.416440$

Sum of electronic and thermal Free Energies= $-2471.500068$

TS3

Zero-point correction= 0.216849 (Hartree/Particle)

Thermal correction to Energy= 0.235138

Thermal correction to Enthalpy= 0.236082

Thermal correction to Gibbs Free Energy=

0.167989

Sum of electronic and zero-point Energies= $-1649.600889$

Sum of electronic and thermal Energies= $-1649.582599$ 
Sum of electronic and thermal Free Energies $=\quad-1649.649749$

TS4

Zero-point correction= 0.216621 (Hartree/Particle)

Thermal correction to Energy= 0.235527

Thermal correction to Enthalpy= 0.236471

Thermal correction to Gibbs Free Energy= 0.166115

Sum of electronic and zero-point Energies= $-1649.601772$

Sum of electronic and thermal Energies= $-1649.582865$

Sum of electronic and thermal Enthalpies= $-1649.581921$

Sum of electronic and thermal Free Energies= $-1649.652278$

TS5

Zero-point correction= 0.215296 (Hartree/Particle)

Thermal correction to Energy= 0.234629

Thermal correction to Enthalpy= 0.235573

Thermal correction to Gibbs Free Energy=

0.163828

Sum of electronic and zero-point Energies= $-1649.604952$

Sum of electronic and thermal Energies= $-1649.585619$

Sum of electronic and thermal Enthalpies= $-1649.584675$

Sum of electronic and thermal Free Energies= $-1649.656421$ 